aus: Enzyklopädie der Neuzeit, hrsg. von Friedrich Jäger, Bd 2 (Stuttgart/Weimar: Metzler, 2005), Sp. 664-681

\title{
Chemische Wissenschaften
}

1. Kenntnisse und Theorien

2. Chemie als Disziplin

3. Apparate und Verfahren

\section{Kenntnisse und Theorien}

1.1. Überblick

1.2. Die montanistische, iatrochemische und

naturphilosophische Tradition

1.3. Von der pneumatischen Chemie

zur Chemischen Revolution

1.4. Atomismus und die Lehre

von der chemischen Verbindung

1.5. Anfänge der Organischen Chemie

\section{1. Überblick}

Unter »Chemie« (etymologisch evtl. aus ägypt. kmt, der Bezeichnung für die schwarze Erde Ägyptens, oder von griech. chēmeía oder chymeía, "Metallguss«, hergeleitet) lassen sich unterschiedliche, auf die Herstellung, Reinigung oder Umwandlung von Stoffen gerichtete Verfahren und Wissensbestände zusammenfassen. Ihren 
Ursprung haben sie in der $\gg$ Metallurgie, der Bereitung von $\gg$ Farbstoffen und $\nearrow$ Glas, der Arzneiherstellung und der Lebensmitteltechnologie. Von der $\rtimes$ Alchemie zunächst auch begrifflich nicht unterschieden, verbanden sich diese Wissensbestände in der Frühen Nz. mit $\gg \mathrm{Pa}-$ racelsismus und $\$ Naturphilosophie $\mathrm{zu}$ einer kosmologisch eingebetteten $\gg$ Materie-Lehre, aus der sich mit eher pragmatischer Zielsetzung und Anknüpfung an die $\gg$ Pharmazie ein Universitätsfach entwickelte.

Die zunächst als Hilfswissenschaft der $\uparrow$ Medizin verstandene Chemie (=CH.) nahm im 18. Jh. engere Verbindungen zur gewerblichen Praxis, dem $\pi$ chemischen Gewerbe sowie zur $\gg$ Naturgeschichte auf. Als Grundlagenwissenschaft für die Erklärung der allgemeinen Eigenschaften von Körpern konnte sich die CH. um 1800 als eigenständige Wissenschaft etablieren, nachdem ihr das 18. Jh. neue Institutionalisierungsoptionen, den theoretischen Rahmen, das analytische Forschungsprogramm und eine systematische $\Uparrow$ Nomenklatur gegeben hatte. Erfolgreiche Akademisierung und ein im pharmazeutisch-gewerblichen Bereich entstehender Arbeitsmarkt wiesen dem Fach im 19. Jh. eine Vorreiterrolle unter den experimentellen $\$ Naturwissenschaften $\mathrm{zu}$. Von der CH. ging die Transformation der $\Uparrow$ Universitäten in arbeitsteilige Forschungsstätten aus, und aus ihr heraus entstand seit der Mitte des 19. Jh.s ein neuer Typus von wissenschaftsbasierter $\nearrow$ Industrie.

\subsection{Die montanistische, iatrochemische und naturphilosophische Tradition}

Nachdem in der Mitte des 16. Jh.s gelehrte Praktiker wie Vannoccio Biringuccio in De la pirotechnia libri $X$ (Venedig 1540; »10 Bücher über die Feuertechnik«) oder Humanisten wie Georgius Agricola in De re metallica libri XII (Basel 1556; dt. »Vom Bergwerck 12 Bücher«, 1557) das chemische (= chem.), hüttenkundliche und montanistische Erfahrungswissen ihrer Zeit samt den praktizierten Verfahren und Hilfsmitteln systematisiert hatten ( $\gg$ Montanwesen), lieferte der $\nearrow$ Paracelsismus den theoretischen Rahmen einer sowohl kosmologisch wie auch theologisch eingebetteten chem. Naturphilosophie [13]. Mit Theoremen wie der Mikrokosmos-Makrokosmos-Analogie, den drei Prinzipien »Salz«, "Schwefel« und »Quecksilber« oder der Lehre von den Signaturen (den äußeren Erkennungszeichen innerer Wirkkräfte) hatte Paracelsus ein ebenso spekulatives wie faszinierendes Programm entworfen. Darin war die körperliche Welt als ein großer chem. Prozess begriffen, was dem Wissenden die Mittel zum praktischen Eingreifen gab ( $/$ Naturmagie).

Die Herausforderung dieses Programms galt den etablierten $\nearrow W i s s e n s s y s t e m e n:(1)$ der Theologie, deren tradiertem $\rtimes$ Dogma Paracelsus einen direkten, magisch- naturmystischen Zugang zur Wahrheit gegenüberstellte; (2) der in der Medizin vorherrschenden $`$ Humoralpathologie, die $\uparrow$ Gesundheit und $\uparrow$ Krankheit aus der Mischung der vier Körpersäfte (Blut, Schleim, gelbe Galle, schwarze Galle) erklärte und der Paracelsus ein völlig anderes Therapiekonzept (nämlich die Gabe mineralisch-chem. Heilmittel) entgegensetzte; (3) schließlich der aristotelischen $\Uparrow$ Naturphilosophie, deren begrifflich-logische Ordnung die $\nearrow$ Universitäten nach wie vor dominierte. Auf den Konflikt zwischen individualistisch-naturmystischer Erfahrung und didaktisch-methodischer Ordnung, der zwischen dem Paracelsisten Oswald Croll und dem Schulmann Andreas Libavius ausgetragen wurde, datiert die neuere Forschung den Ursprung der $\mathrm{CH}$. als einer wiss. $\gg$ Disziplin [16]. Libavius' Alchemia (Frankfurt/Main 1597) systematisierte die chem. Kenntnisse durch logisch-begriffliche Ordnung der Verfahren (Lösung, Destillation, Sublimation) und Apparaturen (Öfen, Gefäße) sowie der Substanzen und ihrer Eigenschaften. Erst in dieser »disziplinierten « Form konnte die paracelsische $\mathrm{CH}$. als Chemiatrie oder \Iatrochemie die Schwelle zur Universitätswissenschaft nehmen, d.h. als eine auf das Verständnis physiologischer Vorgänge und die Bereitung chem. Arzneimittel gerichtete Hilfswissenschaft der $\gg$ Medizin.

Dabei konkurrierten im 17. Jh. zwei unterschiedliche Auffassungen von wiss. CH.: zum einen das iatrochem. inspirierte Programm einer chemia medica, zu deren wichtigsten Exponenten der fläm. Arzt Johann Baptist van Helmont (1577-1644) mit seinem Ortus medicinae (Amsterdam 1648; dt. Aufgang der Artzney-Kunst, Sulzbach 1683) ebenso zählt wie Jean Beguin mit dem Tyrocinium chymicum (Paris 1610; "Chem. Lehrstück «) oder Nicolas Lemery, dessen Cours de chymie (Paris 1673) für viele Generationen das maßgebliche Lehrbuch blieb. In Konkurrenz dazu stand das Programm einer chemia physica (physischen $\mathrm{CH}$.), wie es v.a. Robert Boyle in Oxford entworfen hatte, um die $\mathrm{CH}$. im Zuge des wiederbelebten $\nearrow$ Atomismus als eine Art Mikromechanik in das mechanistische Weltbild der Zeit zu integrieren [10]. Da Boyle alle Substanzeigenschaften durch in komplexen Strukturen konfigurierte Atome einer eigenschaftslosen Universalmaterie (universal matter) ersetzen wollte und daher sogar die Transmutation aller Stoffe ineinander für möglich hielt, erwies sich sein radikaler Reduktivismus in den Ch. W., die auf qualitative Bestimmungen nicht verzichten können, letztlich als Sackgasse. Als »Vater der modernen $\mathrm{CH}$.«, wie man Boyle früher gern genannt hat, kann er nach neuerem Forschungsstand keinesfalls gelten.

In der Folge setzten sich zumeist eklektische Systeme aus paracelsischer Drei-Prinzipien- und aristotelischer Vier-Elemente-Lehre durch, nach denen man die Stoffe in flüchtige "spiritus«, verbrennliche »Öle«, destillierbare 
»Wässer«, lösliche »Salze« und unlösliche »Erden« einteilte. In der Leidener iatromechanischen Schule verband sich dieser Ansatz mit der v.a. von Hermann Boerhaave (1668-1738) vertretenen Auffassung, dass ein nicht näher spezifiziertes Konzept von »Korpuskeln« als den kleinsten qualitativ bestimmten Stoffteilchen die naturwiss. Grundlage für eine rationale $\gg$ Physiologie und Pathologie abgeben könne. In diese Tradition einer von Newton inspirierten korpuskularen Materielehre, die sich als physikalische Ursachenforschung verstand und nicht als praktische Kunst wie in der metallurgischen oder pharmazeutischen Tradition, gehörte auch eine chemica physica, wie sie Michail Lomonossow (1711-1765) an der Akademie von St. Petersburg vertrat.

So stellten sich die Ch. W. um die Mitte des 18. Jh.s als Nebeneinander weithin noch unverbundener Praxisund Wissensfelder dar. Wissenschaft im Sinne eines einheitlichen, widerspruchsfreien und disziplinär organisierten Lehrsystems waren sie nicht. Das Erfahrungswissen von Berg- und Hüttenleuten hatte in der chem. Analyse von Mineralien, Erzen und Legierungen eine beachtliche Höhe erreicht. In der medizinischen Chemie stand die vitalistische Auffassung von Lebensvorgängen ( Vitalismus) neben korpuskularmechanischen Ansätzen, und die Apotheker stellten chem. Präparate und durch chem. Prozeduren gewonnene pflanzliche Wirkstoffe als Bestandteil des Arzneischatzes her.

Die zunehmende Einbindung der Ch.W. in die Ausbildung von Ärzten, Apothekern und Hüttenleuten hatte zur Folge, dass die alte naturphilosophische Frage nach dem Wesen von Substanz und Materie allmählich einem CH.-Unterricht wich, der vornehmlich auf gewerbliche Anwendung zielte. Die Hinwendung zur Praxis brachte dem Fach öffentliche Anerkennung und staatliche Förderung; eine Verwissenschaftlichung industrieller Verfahren setzte jedoch erst im letzten Drittel des 18. Jh.s ein. Frankreich, wo die Verbindung von Wissenschaft, Wirtschaft und Staat seit der Revolution bes. eng war, übernahm dabei die führende Rolle. Die Herstellung von Soda und Schwefelsäure sowie die Chlorbleicherei ( $/$ Bleichereigewerbe) sind frühe Beispiele einer chem. Großindustrie [28]. Auch die $\nearrow$ Laboratorien der staatlichen Münzämter, der Salpeter- und Pulverfabrikation, der Berg- und Hüttenadministration sowie der $\gg$ Porzellan-Manufakturen waren um 1800 wichtige Zentren praktisch-chem. Forschung.

\subsection{Von der pneumatischen Chemie zur Chemischen Revolution}

Die Entwicklung des theoretischen Lehrgebäudes ging zumeist eigene Wege. Die erste übergreifende chem. Theorie, die diesen Namen verdient, stammte von dem Hallenser Mediziner Georg Ernst Stahl (1660-1734). Da- nach sollten Verkalkung (Oxidation) und die Gewinnung von $\gg$ Metallen aus ihren Erzen oder auch die Beziehungen zwischen Schwefel, sulfidischen Erzen und Vitriolen ihre Erklärung in der Annahme finden, dass bei solchen Umsetzungen ein stoffliches Prinzip der Brennbarkeit, das sog. Phlogiston, ausgetauscht werde. Weil das Feuer als wichtigstes instrumentum der $\mathrm{CH}$. galt und chem. Umsetzungen oft unter Wärmeentwicklung verlaufen, konnte die Phlogistontheorie [30] eine große Zahl zuvor zusammenhangloser Phänomene einheitlich erklären. Sie bewährte sich v.a. in der $\gg$ Metallurgie, wurde aber nie zu einem geschlossenen System ausgebaut. Wie die meisten frühen Theorien der $\mathrm{CH}$. bezog sie sich nur auf bestimmte Substratklassen und Reaktionstypen.

Unabhängig davon und eher vom Bemühen um qualitativ-naturhistorische Klassifikation geprägt ( $/ \mathrm{Na}-$ turgeschichte), konnte sich insbes. die $\mathrm{CH}$. der ${ }^{\top}$ Salze zu einem ökonomisch wichtigen Forschungsgebiet entwickeln. Auch die chem. TMineralogie, die v.a. in den skand. Ländern florierte und in dem schwed. Chemiker Torbern Bergman in Uppsala ihren bedeutendsten Vertreter besaß, blieb dem naturhistorischen Paradigma verpflichtet. Andere Forscher hingegen versuchten, inspiriert von der Newton'schen Physik ( Newtonianismus), die $\mathrm{CH}$. als quantifizierende Wissenschaft von Teilchen und den zwischen diesen wirkenden Kräften neu zu formulieren ( $\uparrow$ Affinität), um das Fach von dem Makel einer bloßen Experimentierkunst zu befreien.

Beim Experimentieren ( $\$ Experiment) in fester und flüssiger Phase hatte man gasförmige Reaktionsprodukte, die sich nicht zu Flüssigkeiten kondensieren ließen, in der Regel als gewöhnliche Luft angesehen und nicht weiter beachtet. Die Beobachtung des engl. Geistlichen Stephen Hales, dass Luft sich »fixieren« und aus Pflanzen oder Mineralien wieder freisetzen lasse, sowie die Entdeckung des schott. Chemikers Joseph Black von 1756, dass sich aus der - bis dahin für einen reinen Stoff oder Element gehaltenen - Luft ein von atmosphärischer Luft verschiedener Bestandteil als »fixe Luft« (unser $\mathrm{CO}_{2}$ ) abtrennen lässt, gaben den Anstoß zu einer bis dahin unbekannten $\mathrm{CH}$. der Gase ("pneumatische $\mathrm{CH}$.«, 7 Gaschemie), die neuartige apparative Maßnahmen forderte (s.u. 3.4.). 1774/75 erhielt der engl. Naturforscher Joseph Priestley bei dem Versuch, ohne Holzkohle metallisches Quecksilber aus seinen Erzen darzustellen, eine neue und »überaus atembare« (eminently respirable) Art von »Luft«. Er nannte sie dephlogisticated air, weil er glaubte, der atmosphärischen Luft das Phlogiston entzogen zu haben.

Um 1772 begann Antoine Laurent de Lavoisier an der Pariser Académie des Sciences mit Untersuchungen zur Fixierung und Freisetzung von »Luft». Die dabei beobachteten Gewichtsänderungen brachten ihn auf den Ge- 
danken, solche Prozesse im geschlossenen System mit Hilfe der Waage (s.u. 3.3.) systematisch zu bilanzieren ( $/$ Messung und Quantifizierung). Die neuere Forschung hat seine Methode mit der Praxis kaufmännischer Buchhaltung und der ökonomischen Theorie seiner Zeit in Verbindung gebracht ( $/$ Physiokratie) und als den eigentlichen Beginn der modernen, quantitativen $\mathrm{CH}$. bezeichnet [18]. Auf dieser Grundlage entwickelte Lavoisier 1777 eine neue Theorie der Verbrennung, bei der ein Wärmestoff (franz. calorique, 7 Wärmelehre) an die Stelle des Phlogistons und an die Stelle von Priestleys »dephlogistierter Luft" der Sauerstoff trat, den Lavoisier als das für die saure Eigenschaft der 7 Säuren verantwortliche Prinzip ansah.

Damit blieb Lavoisiers Reform zwar einerseits der alten Substanzmetaphysik und der Lehre von den Imponderabilien verpflichtet, wies aber zugleich einen Weg, das chem. Wissen der Zeit von einem zentralen Gesichtspunkt her neu zu ordnen. Begleitet wurde dies von einer grundlegenden Reform der chem. Nomenklatur, die sich an der Systematik Linnés orientierte ( $/$ Botanik), und der Publikation seines Lehrbuchs Traité élémentaire de chimie (Paris 1789). In diesem ist das neue, analytisch definierte Konzept des chem. TElements und die Auffassung von der $\mathrm{CH}$. als quantitativanalytischer Naturwissenschaft programmatisch entfaltet. Lavoisiers Schüler, allen voran Guyton de Morveau, benutzten die bereits 1750 in Diderots und D'Alemberts Encyclopédie beschworene Idee einer längst überfälligen »Revolution in der CH.« [4] - unter bewusster Verwendung des politisch konnotierten Begriffs - geschickt, um die neue, "antiphlogistische« $\mathrm{CH}$. zu propagieren [15]. Gegen den heftigen Widerstand brit. und vor allem dt. Naturforscher, der mit der gleichzeitig einsetzenden Herausbildung nationaler Wissenschaftlergemeinschaften einherging [20], setzte sich die »franz.« $\mathrm{CH}$. in den 179oer Jahren rasch in ganz Europa durch [7].

\subsection{Atomismus und die Lehre von der chemischen Verbindung}

Das analytische Forschungsprogramm der $\mathrm{CH}$. ging davon aus, dass sich $\nwarrow$ Elemente in festen Zahlenverhältnissen verbinden und Verbindungen sich auf diesem Wege eindeutig definieren lassen. Bei Mineralien ist dies aber nicht immer der Fall. Die von 1801 bis 1807 zwischen Claude-Louis Berthollet in Paris und JosephLouis Proust in Madrid geführte Kontroverse um das von Proust aufgestellte »Prinzip der konstanten Sättigungsproportionen « war deshalb so lange nicht zu entscheiden, wie nicht klar war, was die elementaren Einheiten einer quantifizierenden $\mathrm{CH}$. sind. Die Vollendung der »Chem. Revolution« gelang daher erst einem Außenseiter, und zwar durch Rückgriff auf den $`$ Atomis- mus Newton'scher Prägung: Zur Deutung der Löslichkeit von Gasen in Wasser hatte John Dalton (1766-1844) in Manchester angenommen, dass die Materie aus unveränderlichen, kugelförmigen Atomen bestehe, jedes chem. Element aber genau eine Art von Atomen besäße und sich v.a. durch deren charakteristische Masse auszeichnete. Durch die Verbindung des Lavoisier'schen Elementbegriffs mit der Atomtheorie lieferte Daltons $A$ New Theory of Chemical Philosophy (London 1808) eine Theorie der chem. Zusammensetzung, die sich erfolgreich operationalisieren ließ.

Denn mit der Atommasse besaß der Experimentator eine Messgröße, die mit den gravimetrischen Verfahren der Zeit hinreichend genau zu bestimmen war. Da die Atommasse eines Elements auch in dessen Verbindungen konstant bleibt, ergibt sich das Verbindungsgewicht aus der Summe der Atomgewichte der Elemente. Weiter nahm Dalton an, dass (1) Elemente einatomig seien; (2) wenn aus zwei Elementen nur eine Art von Verbindung erhalten wird, diese zweiatomig sei; und (3) wenn sich aus zwei Elementen unterschiedliche Verbindungen erhalten lassen, deren Bestandteile in ganzzahligen Verhältnissen stünden. Bezog man nun die relativen Atommassen auf den Wasserstoff mit der Einheit 1, so ergaben sich nicht nur höchst einfache Zahlenverhältnisse, sondern es ließen sich auch die von Proust als empirische Regel gefundenen ganzzahligen Proportionen als fundamentales Naturgesetz begründen. Nicht zu Unrecht verglich ein Zeitgenosse die Leistung Daltons mit der Kepler'schen Wende in der $\uparrow$ Astronomie [1. Bd. 7, 95].

Auch wenn der bildhaft-realistische Atomismus Daltons sich nicht durchsetzen konnte und man in den Ch. W. bis weit ins 19. Jh. hinein pragmatisch am Äquivalentgewicht als bloßer Recheneinheit festhielt [27], so gab die Atomtheorie doch die Grundlage ab für die von Jöns Jakob Berzelius (1779-1848) in Stockholm entwickelte, im Wesentlichen noch heute gültige chem. Zeichensprache ( $\$ Symbole, chemische). In dieser bezeichnen die Anfangsbuchstaben der lat. Elementnamen zugleich Masseneinheiten, sodass sich chem. Reaktionen als quantitative Gleichungen aufschreiben lassen [12].

Weder Lavoisier noch Dalton hatten die Natur der chem. Bindung plausibel erklären können, und das Konzept der chem. \Affinität war durch die Arbeiten Berthollets problematisch geworden. Da stellte die 1800 in London publizierte Entdeckung des ital. Physikers Alessandro Volta, dass sich durch aufeinander geschichtete Plattenpaare zweier Metalle Strom erzeugen lässt ( $/ E l e k-$ trizität), die chem. Bindungslehre auf eine neue theoretische Basis. Weil elektrische Prozesse mit chem. Umsetzungen verbunden sind, führte der $\uparrow$ Galvanismus zu der Auffassung, dass die Anziehung zwischen den Elementen und die Bildung elektrisch neutraler Verbindungen von elektrischen Kräften bewirkt wurden. Mit Hilfe 
Voltaischer Batterien konnte Humphry Davy ab 1807 an der Royal Institution in London aus den bis dahin für elementar angesehenen "Erden« die Metalle Natrium, Kalium, Magnesium, Calcium, Strontium und Barium gewinnen. Sein Schüler Michael Faraday (1791-1867) zeigte darüber hinaus, dass bei elektrochem. Reaktionen feste Beziehungen zwischen chem. Masse und Ladungsmenge existieren. Von Berzelius fortentwickelt und durch sein aus dem Schwedischen übersetztes Lehrbuch der Chemie (dt. 1820-1831 u.ö., franz. 1829-1833) in ganz Europa verbreitet, lieferte die »dualistische Theorie«, in Verbindung mit Atomtheorie und der Berzelius'schen Zeichensprache, die für die erste Hälfte des 19. Jh.s wichtigste Theorie der $\mathrm{CH}$. Zugleich wurde sie die Basis der später auch industriell wichtigen Elektrochemie.

\subsection{Anfänge der Organischen Chemie}

Die Übertragung dieses Ansatzes auf Stoffe aus dem Tier- und Pflanzenreich führte allerdings zu Problemen. Schon Lavoisier hatte nachgewiesen, dass organische Verbindungen aus einer sehr kleinen Zahl von `Elementen, nämlich Kohlenstoff, Wasserstoff, oft auch Sauerstoff und Stickstoff, aufgebaut und damit den Methoden der quantitativen Analyse zugänglich sind. Die durch Berzelius begründete und von Justus Liebig (1803-1873) gemeinsam mit Friedrich Wöhler experimentell bestätigte sog. Radikaltheorie nahm an, dass in organischen Verbindungen Kohlenwasserstoffgruppen (»Radikale«) die Rolle der Metallatome in mineralischen Stoffen übernehmen, bei Umsetzungen als Gruppen erhalten bleiben und dass auch hier elektrische Kräfte die Bindung bewirken. Das Problem des 7 Vitalismus, d.h. die Annahme, dass physiologische Prozesse von einer bes. Lebenskraft bewirkt seien, war für die Chemiker dabei irrelevant. Wöhlers berühmte Harnstoffsynthese von 1828 , bei der er einen vorgeblich "anorganischen« Stoff in das »organische "Stoffwechselprodukt Harnstoff verwandelte, ist oft als Widerlegung der Lebenskraft missverstanden worden [26].

Substitutionsreaktionen hatten jedoch deutlich gemacht, dass die elektrochem. Natur solcher "Radikale keine definierte Stoffeigenschaft ist; das 1830 entdeckte Phänomen der Isomerie, wonach unterschiedliche Verbindungen die gleiche Summenformel besitzen können, lenkte die Aufmerksamkeit auf die Frage nach der chem. »Konstitution«, d.h. der innermolekularen Gruppierung der Elemente. Dies ist der eigentliche Beginn der Organischen CH. - ein Begriff, den Novalis im Geist der Romantischen $\gg$ Naturphilosophie einführte und den Hermann Kolbe auf die CH. der Kohlenstoffverbindungen eingrenzte [2. Bd.1, 4]. Kolbe war Exponent einer Gruppe junger Forscher, die um 1845 das von Lavoisier geprägte analytische Programm der $\mathrm{CH}$. für erschöpft hielten und sich am Beginn einer neuen Ära der synthetischen $\mathrm{CH}$. sahen. Sie hielten es nämlich für möglich, nun auch komplizierte Naturstoffe künstlich zusammenzusetzen, ja die Natur durch synthetische Substanzen zu übertreffen. In der Tat war Kolbe 1845 die erste vollständige Synthese eines Naturstoffs, der Essigsäure, aus den Elementen gelungen, und in den 1850er Jahren entstanden die ersten synthetischen Farbstoffe. Sie leiteten den spektakulären Aufstieg der Farbenindustrie ein, mit der die dt. Chem. Industrie um 1900 den Weltmarkt beherrschen sollte.

\section{Chemie als Disziplin}

2.1. Konkurrierende Ansätze

2.2. Hilfswissenschaft der Medizin

2.3. Neubewertung als Grundlagenwissenschaft

2.4. Ausbildung, Kommunikation, Disziplingenese

\subsection{Konkurrierende Ansätze}

Während im dt. und skand. Raum chem. Wissen zur Ausbildung von Apothekern, Ärzten, Mineralogen oder Metallurgen gehörte, galten die Ch. W. in Paris eher als Teil der allgemeinen, experimentellen Naturwissenschaft ( Physikalische Wissenschaften), wie sie etwa an der Académie des Sciences auf höchstem Niveau betrieben wurde. Dank öffentlicher Vorlesungen z. B. am Jardin $d u$ Roi, wo Diderot und Rousseau zu den Hörern zählten, genoss die $\mathrm{CH}$. in Frankreich großes Prestige. In England hingegen fehlten chem. Ausbildungsstätten bis zur Mitte des 19. Jh.s. Stattdessen machten freie Demonstratoren die $\mathrm{CH}$. dort im 18. Jh. zum festen Bestandteil einer öffentlichen Experimentalkultur [14]. Doch so heterogen wie die Wissens- und Praxisfelder der frühen $\mathrm{CH}$. war auch das Spektrum ihrer Institutionen: $\nearrow$ Universität und $\nearrow$ Apotheke, Probierkammer und $\lceil$ Akademie, Münze und $\nearrow$ Medizin, Bergwerk ( $`$ Bergbautechnik) und Färberei ( $\lambda$ Farbstoffe) boten ganz unterschiedliche soziale und intellektuelle Bedingungen. Besäßen wir heute nicht einen einheitlichen Begriff von $\mathrm{CH}$., so erschienen uns diese ganz unterschiedlichen "Chemien« jener Zeit wohl kaum als zusammengehörig.

Allein das $\gg$ Laboratorium als der charakteristische Ort dieser Praktiken und das zum Labor gehörige Ensemble seltsamer Gerätschaften und Öfen erklären, dass es seit der Frühen Nz. eine - ikonographisch reich belegte - relativ einheitliche Wahrnehmungstradition gab. Aus dieser tritt uns die $\mathrm{CH}$. meist als Inbegriff vergeblichen Strebens, das Laboratorium als Ort der Unordnung, der Chemiker oder Alchemist als »betrogener Betrüger « entgegen ( 7 Alchemie) [17].

Ein Bewusstsein fachlich-wiss. Eigenständigkeit bildete sich zunächst im Bereich des chem. Hochschulfaches heraus. Denn als die wichtigste Differenzierungs- 
form der nzl. Wissenschaft sorgen die $`$ Disziplinen für Selektion und Qualifikation, Innovation und Stabilisierung, Tradition und Legitimation wissenschaftlichen Wissens ( $/ W i s s e n$ und Wissensideale). Disziplingenese lässt sich dabei als Ergebnis eines kollektiven Prozesses begreifen, der unter Anpassung an eine sich wandelnde Umgebung jeweils neue soziale Strukturen schafft und diese institutionell stabilisiert. Dabei konkurrieren auf jeder Stufe unterschiedliche Forschungsprogramme, Handlungsnormen und Institutionalisierungsstrategien miteinander ( 7 Wissensorganisation) [24].

\subsection{Hilfswissenschaft der Medizin}

Bereits 1609 hatte der natur- und okkultwiss. interessierte Landgraf Moritz von Hessen-Kassel in Marburg seinen Leibarzt Johann Hartmann, einen Anhänger des TParacelsismus, zum chymiatriae professor publicus und damit auf den ersten Universitätslehrstuhl für ein chem. Fach berufen. Im Lauf des 17. Jh.s konnte die $\mathrm{CH}$. dann an vielen europ. Universitäten Fuß fassen, wurde dabei aber - in Abkehr vom ursprünglichen Programm einer kosmisch begründeten chem. Philosophie - mit praktisch-pharmazeutischer Zielsetzung in den Lehrplan der 7 Medizin eingebunden. Dies befreite sie aus dem Verdacht alchemischer Obskurität sowie aus dem starren Theorierahmen der Naturphilosophie und bot die Chance praktischer Bewährung, was mit einem Prestigezuwachs einherging. Da das Fach innerhalb der ${ }^{\top}$ Medizinischen Fakultäten jedoch meist vom rangniedrigsten $\lambda$ Professor in Verbindung mit ${ }^{\top}$ Anatomie, $\lambda$ Botanik und Arzneimittellehre ( $/$ Pharmazie) vertreten wurde, blieb es auf seine hilfswiss. Funktion beschränkt. Fachprofessuren waren bis ins ausgehende 18. Jh. die Ausnahme; man rückte vielmehr nach Anciennität auf freiwerdende "höhere" Stellen vor, und so bot das System wenig Anreiz, sich auf eine der Hilfswissenschaften zu verlegen. Versuche zur Abschaffung dieses "Aufrückens«, wie sie etwa in der Habsburgermonarchie unternommen wurden, scheiterten meist am geringen Differenzierungsgrad der frühnzl. Universität. Oft war die CH.-Professur daher nicht viel mehr als der Einstieg in eine medizinische Karriere.

\subsection{Neubewertung als Grundlagenwissenschaft}

Aus diesem Dilemma heraus entstanden zu Beginn des 18. Jh.s unterschiedliche Institutionalisierungsstränge, die miteinander um akademische Ressourcen wetteiferten. Es ging um die programmatische Umwertung von der Hilfswissenschaft zur Grundlagenwissenschaft. Das franz. Modell, die CH. als allgemeine Naturwissenschaft mit Physik ( 7 Physikalische Wissenschaften) zu verbinden, wie es in der Pariser Académie des Sciences bereits im 17. Jh. geschehen war, kulminierte 1794 mit Gründung der $\nearrow$ École Polytechnique, einem europaweit einmaligen Zentrum chem. Exzellenz und systematischer Laboratoriumsausbildung. Außerhalb der differenzierten und klar hierarchisierten Wissenschaftslandschaft der franz. Hauptstadt wäre ein solches Modell jedoch nicht zu realisieren gewesen. Größere Breitenwirkung entfaltete deshalb der Versuch der iatromechanischen Schule, von der korpuskular aufgefassten $\mathrm{CH}$. her eine rationale $\gg$ Physiologie und Pathologie ( $/$ Krankheit) zu begründen. Damit wurden chem. Kenntnisse auch für den klinisch tätigen Arzt zunehmend wichtig.

Parallel dazu verlief ein weiterer Institutionalisierungsstrang, der die Ch.W. stärker auf ihre gewerblichen Anwendungen hin orientierte ( $T$ Chemische Gewerbe), um sie aus der Anbindung an die Medizin zu lösen. Der schwed. Chemiker Johan Gottschalk Wallerius unterschied in diesem Zusammenhang 1751 erstmals zwischen "reiner « und "angewandter « Wissenschaft, um den diskriminierenden Gegensatz von »theoretischer Wissenschaft» und "praktischer Kunst« zu überwinden und die $\mathrm{CH}$. vom Makel des bloß Handwerklichen zu befreien [5]. Indem er sie mit dem $\nearrow$ Utilitarismus der Zeit und einem gewandelten Verständnis von $\nearrow$ Öffentlichkeit verband, war ein neuer Orientierungsrahmen geschaffen, der den außermedizinischen, ökonomisch-gesellschaftlichen Bezügen der Ch.W. eine Schlüsselfunktion zuwies [23]. Neben der medizinischen $\mathrm{CH}$. nannte Wallerius mineralogische $\mathrm{CH}$. (lat. chemia lithurgica), Salz- (chemia halurgica), Feuer- (chemia thejurgica), Metall-, Glas-, Agrikultur- (chemia oeconomica), Farb(chemia chromatica) und Kunst- oder Handwerks-CH. (chemia technica, opificiaria) als die wichtigsten Gegenstandsbereiche.

An den dt.sprachigen Hochschulen, die diesem Modell folgten, kam es im letzten Drittel des 18. Jh.s zu einer engen Verbindung von CH. und Kameralwissenschaften, wobei erstere seitens der sich gerade erst institutionalisierenden Kameralistik wirksame Unterstützung erfuhr. Der $\nearrow$ Kameralismus ordnete die $\mathrm{CH}$. den wirtschaftsund ordnungspolitischen Zielsetzungen des Staates zu, legitimierte damit ihren Anspruch auf gesellschaftliche Ressourcen und verwies zugleich auf das Rationalisierungs- und Modernisierungspotential experimenteller $\lceil$ Naturwissenschaft. Die institutionellen Folgen dieses Disziplinwandels kamen v.a. dort zur Geltung, wo die CH.-Professur nicht primär der Ausbildung künftiger Ärzte diente. Modellcharakter hatten in dieser Hinsicht die schwed. Universitäten Uppsala, Lund und Åbo, wo die $\mathrm{CH}$. zu den ökonomischen Fächern gehörte.

Nicht zu unterschätzen ist auch die Rolle der im letzten Drittel des 18. Jh.s entstandenen $\gg$ Bergakademien, wo sich die ersten Fachvertreter nachweisen lassen, die keine medizinische Ausbildung durchlaufen 
hatten. Universitätsprofessuren für $\mathrm{CH}$. in Verbindung mit Technologie oder "Ökonomie» (d.h. `Landwirtschaft) sind eine charakteristische Erscheinung der Zeit zwischen 1775 und 1820; aber auch im $\nearrow$ Laboratorium der Preuß. Akademie der Wissenschaften in Berlin, dem bestausgestatteten Deutschlands, arbeiteten Johann Heinrich Pott und Andreas Sigismund Marggraf über Fragen der Erschließung und Verarbeitung heimischer Rohstoffe. Obwohl das Beckmann'sche Programm einer akademischen Technologie letztlich scheiterte und die Kameralwissenschaften im ersten Drittel des 19. Jh.s auseinander brachen, war damit ein tragfähiger Grund für eine Fortentwicklung der Ch.W. außerhalb des medizinischen Curriculums gelegt.

\subsection{Ausbildung, Kommunikation, Disziplingenese}

In der Zwischenzeit hatte eine weitere Institutionalisierungsstrategie an Bedeutung gewonnen. Im späten 18. Jh. galt nämlich die traditionelle Apothekerausbildung in Form eines Lehrverhältnisses als fachlich unzulänglich und den gesellschaftlichen Ambitionen des Berufsstandes hinderlich ( $/$ Apotheke). Akademisierung sollte hier Abhilfe schaffen. Dem Beispiel von Johann Christian Wiegleb in Langensalza sowie Johannes Bartholomäus Trommsdorff in Erfurt folgend, entstanden in Deutschland seit 1779 private Lehranstalten, in denen Pharmazeuten, Drogisten und Gewerbetreibende eine praktisch-wiss. Ausbildung erhalten sollten. Da von 1805 an in den meisten dt. Ländern Apotheker ein vorbereitendes Universitätsstudium zu absolvieren hatten, stellte die $\$ Pharmazie das wichtigste soziale Reservoir dar, aus dem sich der entstehende Chemikerberuf rekrutierte - lange bevor im Bereich der $\$ Chemischen Gewerbe, der einsetzenden $\nearrow$ Industrialisierung oder der Landwirtschaft ( $\$ Agrikulturchemie) ein eigentlicher Bedarf an akademisch oder auf polytechnischen Schulen ausgebildeten Chemikern existierte [19]; [22].

In diesem Umfeld vollzog sich die entscheidende Phase der Herausbildung der CH. als akademischer Disziplin mit klaren Karrieremustern, Fachprofessuren und festen Kommunikationsstrukturen [20]. Lorenz von Crells Chemisches Journal für die Freunde der Naturlehre, Arzneygelahrtheit, Haushaltungskunst und Manufacturen (Lemgo 1778-1781) ist nicht nur die erste $\rtimes F a c h z e i t s c h r i f t$ der Ch.W., sondern die erste disziplinorientierte naturwiss. Fachzeitschrift überhaupt. Ihr folgten 1789 die von Lavoisier begründeten Annales de chimie, das neben den 1832 von Justus Liebig herausgegebenen Annalen der Chemie und Pharmacie wichtigste Kommunikationsmittel der sich formierenden Disziplin ( $/$ Disziplinen, gelehrte).

Ein weiterer und folgenreicher Institutionalisierungsschub ging von Gießen aus. Dort hatte Liebig, zuvor in Paris in die neuesten Methoden der chem. Forschung eingeführt, 1824 ein kleines »Chem.-pharmaceutisches Institut« gegründet, das nach dem Modell pharmazeutischer Privatinstitute elementare praktischchem. Kenntnisse vermitteln sollte. Seit Mitte der 183oer Jahre entwickelte sich Liebigs Institut zu einer Einrichtung, deren Erfolgsrezept in der forcierten Hinführung zur experimentellen $\nearrow$ Forschung und der arbeitsteiligen Organisation der Forschungspraxis bestand. Liebigs Chemische Briefe, die 1844 zunächst als Artikelserie in der Augsburger Allgemeinen Zeitung, kurz darauf simultan in dt. und engl. Buchausgaben erschienen ( $/$ Popularisierung) und Übersetzungen in fast alle europ. Sprachen erfuhren, stellten die $\mathrm{CH}$. als Zeichenlehre und Universalgrammatik der stofflichen Welt sowie als einen unverzichtbaren Teil der Allgemeinbildung dar. Gleichzeitig aber liefere die auf den Methoden der reinen $\mathrm{CH}$. beruhende chem. Industrie den besten Maßstab für den Reichtum einer Nation.

Studenten und Gastwissenschaftler v.a. aus Frankreich und Großbritannien, die bei Liebig studiert hatten, exportierten das Gießener Konzept seit den 1840er Jahren an andere europ. Hochschulen. Die erfolgreichste unter diesen ausländischen Forschungseinrichtungen war das 1845 gegründete, von Liebigs Starschüler August Wilhelm Hofmann geleitete Royal College of Chemistry in London. Zehn Jahre später hatten Liebigs Schüler praktisch sämtliche $\mathrm{CH}$.-Professuren an den Universitäten, Colleges und Lehrkrankenhäusern des Vereinigten Königreichs besetzt. Andere gründeten oder leiteten Industriebetriebe. Mit dieser breiten und erfolgreichen Institutionalisierung übernahmen die Ch.W. in der zweiten Hälfte des 19. Jh.s eine Führungsrolle bei der Transformation der Hochschulen von Lehr- in Forschungseinrichtungen sowie bei der Entstehung eines neuen Typs wissenschaftsbasierter $\nearrow$ Industrie.

\section{Apparate und Verfahren}

3.1. Chemie als herstellende Wissenschaft

3.2. Destillation und Metallurgie (Probierkunde)

3.3. Quantifizierung und Instrumente

3.4. Gasapparaturen und Elementaranalyse

\subsection{Chemie als herstellende Wissenschaft}

Ort der Ch.W. ist das \Laboratorium, eine Werkstatt, in der neues Wissen geschaffen wird. Dabei entstammen die Stoffe und Mischungen des Chemikers typischerweise nicht dem vorhandenen Repertoire der von Natur aus vorgegebenen Dinge. Damals wie heute muss die $\mathrm{CH}$. die Mehrzahl ihrer Gegenstände erst erzeugen, um sie zum Objekt praktischer und theoretischer Untersuchungen zu machen. In der $\mathrm{CH}$. ist daher der Aspekt der Wissenskonstruktion bes. augenfällig; sie 
ist eine handlungsorientierte, herstellende Wissenschaft. Ihre wichtigsten Hilfsmittel sind Apparate und apparative Verfahren; aber auch Handgriffe und Maßnahmen zur Kontrolle von Temperatur und Zeit gehören dazu.

Wie im gewerblichen Bereich und in den Pharmakopöen der Apotheker erfolgte auch in der $\mathrm{CH}$. die †Standardisierung und Identitätsprüfung lange v.a. mit Hilfe genau zu befolgender Herstellungsanweisungen. Noch Boerhaaves einflussreiche Elementa chemiae (Leiden 1732) präsentieren die $\mathrm{CH}$. als Handlungs- und Verfahrenslehre: Der allgemeine Teil dieses lat. $\nearrow$ Lehrbuchs behandelt die Hilfsmittel, Lösungsmittel und Gerätschaften, der spezielle Teil einzelne Prozesse, jeweils nach Herstellungsverfahren (lat. apparatus) und Anwendungszwecken (lat. usus) systematisiert und typischerweise mit »Man nehme ...« (lat. sumatur) beginnend. Lösungsmittel und Feuer als den Grundoperatoren in den Ch.W. entsprachen die beiden Grundtypen der Apparatur: Gefäße und Öfen.

\subsection{Destillation und Metallurgie (Probierkunde)}

Reinigungsverfahren wie Extraktion, Filtrieren, Sublimation, Kristallisation und Destillation oder auch Reaktionen bewirkende Maßnahmen wie etwa Auflösen, Schmelzen ( $\$ Schmelzprozess), Mischen und Erhitzen spielten seit jeher eine zentrale Rolle für die Praxis der Ch.W. Sie gehen sämtlich auf die Antike zurück und wurden in der $\Upsilon$ Alchemie weiterentwickelt. Herausragende Bedeutung kam dabei der Destillation $\mathrm{zu}$, die im europ. MA so weit vervollkommnet wurde, dass sich damit reiner Alkohol gewinnen ließ. In Form eines pharmazeutisch-technischen Handbuchs wurde dieses Praxiswissen erstmals im Liber de arte destillandi de compositis (1500; dt. 1512) des Straßburger Chirurgen Hieronymus Brunschwig zusammengestellt. Es enthält die detaillierten Beschreibungen und Abbildungen zahlreicher Destillationsgefäße: den Alembic oder Destillationshelm, den Kolben, die Retorte, Rückflussvorrichtungen usw. Mit der alchemischen Auffassung von der Fähigkeit aller Materie, zur Vervollkommnung zu gelangen, und der Aufgabe des Alchemisten, diese in der Natur angelegte Tendenz mit menschlicher Kunst zu bewirken, verband sich die paracelsische Überzeugung, das wirksame Prinzip oder »innere Wesen« der Heilmittel lasse sich als flüchtige Essenz (lat. quinta essentia) gewinnen. Damit besaß das Verfahren der Destillation eine weit über das Praktische hinausweisende Bedeutung ( $\nearrow$ Spiritus).

Die im $\nearrow$ Montanwesen gängigen Verfahren und Gerätschaften sind in den sog. Probierbüchern ( $/$ Probierkunde) des 16. Jh.s sowie in den Kompendien des sächs. Humanisten Georgius Agricola (s.o. 1.2.) oder des böhm. Metallurgen Lazarus Ercker überliefert. Der Traktat De sceuastica artis, den Andreas Libavius der zweiten Auflage seiner Alchemia (Frankfurt/Main 1606) als Handbuch der chem. »Gerätekunde« beigab, enthält eine umfassende Zusammenstellung von Probieröfen, Schmelztiegeln und Destillationsgefäßen [25]. So phantastisch manche dieser Vorrichtungen anmuten, ihre historische Verwendung ist doch inzwischen durch Grabungsbefunde gesichert [29].

\subsection{Quantifizierung und Instrumente}

Die Waage ( 7 Gewichtsmessung) ist seit dem 14. Jh. als Hilfsmittel des Chemikers und Metallurgen bezeugt, in der Arzneibereitung sowie im kommerziellen Bereich; insgesamt reicht ihr Gebrauch noch weiter zurück. Ansätze einer analytisch-quantifizierenden Naturwissenschaft wird man darin jedoch nicht sehen dürfen. Denn bis ins 18. Jh. blieb die $\mathrm{CH}$. der qualitativ-naturhistorischen Methode und der Frage nach der Substanz verpflichtet. Für diese aber galt der aristotelische Grundsatz, dass die Substanz nicht unter die Kategorie der Quantität falle. Zahlenwerte blieben daher ein äußeres Bestimmungsmerkmal: über den Stoff selbst und seine chem. Besonderheit besagten sie nichts. Die $ૉ$ Maße und Gewichte gehörten eher in den kaufmännischen Bereich des Taxierens, und dieser verlangte keine besondere Präzision ( $/$ Messung und Quantifizierung). Dies gilt auch für den Bereich der Probierkunde, deren hochentwickelte analytische Methoden primär der Bestimmung der Abbauwürdigkeit galten und nicht dem naturwiss. Erkenntnisgewinn.

Erst mit dem Grundsatz des Massenerhalts, der implizit bereits in der Antike bekannt war, doch erst von Lavoisier als Naturgesetz formuliert und für die chem. Forschung operationalisiert wurde, sowie mit Lavoisiers Methode der exakten Bilanzierung chem. und physiologischer Prozesse in geschlossenen Systemen (s.o. 1.3.) erhielt die Waage ihre zentrale Bedeutung für die nzl. $\mathrm{CH}$. Mit zunehmenden Anforderungen an die Präzision wurde die Waagentechnologie zu einer der instrumentellen Determinanten in der Entwicklung der analytischen $\mathrm{CH}$., wobei die entscheidenden $T$ Innovationen (kurzarmige und Mikrowaagen) erst in die zweite Hälfte des 19. Jh.s fallen [31].

Die Entwicklung der Apparate des Chemikers, Apothekers und Probierers blieb bis um 1800 bemerkenswert traditionell [3]. Der Grund liegt in der meist geringen Spezifik dieser Hilfsmittel, deren einfache Grundtypen sich unterschiedlichen Zwecken anpassen ließen. Dass der Experimentator diese selbst anfertigte und auch Glasapparaturen selbst blies, war die Regel. Kommerzielle Lieferanten von Laborbedarf kamen erst im frühen 19. Jh. auf; nur Waagen musste man aus den wenigen Werkstätten beziehen, die sich auf die Herstellung so 
komplexer $\rtimes$ wissenschaftlicher Instrumente verstanden. Die wenigen wirklichen Neuerfindungen im chem. Instrumentenbau des 18. Jh.s, wie das beim Probieren von Erzen wichtige Lötrohr, blieben auf enge Anwendungskontexte beschränkt. Die großen Neuerungen in der chem. Laboratoriumstechnik, die sich mit der Einführung des Stadtgases und der `Elektrizität als Energieträger verbinden, fallen erst in die zweite Hälfte des 19. Jh.s.

\subsection{Gasapparaturen und Elementaranalyse}

Die wichtigsten apparativen $\nearrow$ Innovationen der nzl. $\mathrm{CH}$. gingen von der pneumatischen Chemie ( $/ \mathrm{Gas}-$ chemie) aus, die ihrerseits an Techniken anknüpfen konnte, die man seit der zweiten Hälfte des 17. Jh.s beim Experimentieren mit dem $\nearrow$ Vakuum und der Luftpumpe $\mathrm{zu}$ beherrschen gelernt hatte. Probleme der Dichtigkeit und der Verbindung unterschiedlicher Werkstoffe stellten dabei erhebliche Anforderungen an Erfindungsreichtum und handwerkliches Geschick. Der entscheidende Übergang von den schlichten Glasgefäßen und offenen Quecksilbertrögen, mit denen man noch in der Mitte des 18. Jh.s die einzelnen »Luftarten« aufgefangen hatte, zu hoch komplexen Apparaturen aus Stahl, Messing und Glas fand im Forschungsprogramm Lavoisiers statt, das auf das präzise Bilanzieren stofflicher Umsetzungen in hermetisch geschlossenen Systemen zielte. Lavoisiers gasanalytische Apparatur, die heute im Pariser Conservatoire des Arts et Métiers aufbewahrt wird, kostete damals mehr als das Jahresgehalt eines hauptamtlichen Mitglieds der Académie des Sciences, und nur in Paris gab es damals $\nearrow$ Instrumentenmacher, die in der Lage waren, derart komplizierte Apparaturen herzustellen. Durchgesetzt hat sich diese Technik daher erst in der miniaturisierten Form der Eudiometrie oder Luftgütemessung, deren Entwicklung v.a. auf Felice Fontana in Florenz zurückgeht [6].

Bes. folgenreich wurde die Elementaranalyse für die organische Chemie. Dass eine bestimmte Gewebeprobe $12 \%$ Fett, $20 \%$ Schleim, 44\% phosphorsaure Kalkerde, $1 \%$ Eisenoxyd etc. enthalte, wie man es in den medizinisch-chem. Analysen der Zeit liest, half nicht einmal dem Arzt sehr viel weiter. Die üblichen gravimetrischen Verfahren zur Ermittlung der chem. Zusammensetzung wie die Ausfällung als Salz oder Veraschung mit anschließender prozentualer Bestimmung der mineralischen Rückstände waren dem pflanzlichen oder tierischen Untersuchungsmaterial wenig angemessen. Lavoisier hatte deshalb die Isolierung reiner Substanzen und die quantitative Ermittlung der darin enthaltenen Elemente zur wichtigsten Aufgabe der $\mathrm{CH}$. bestimmt. Auch hatte er bereits einen Weg gewiesen, um den Wasserstoff-, Kohlenstoff- und Sauerstoffgehalt einer organischen Substanz volumetrisch, d.h. durch Volumenmes- sung, zu ermitteln. Joseph-Louis Gay-Lussac entwickelte dieses Verfahren weiter und baute es durch den Befund, dass Gasreaktionen in ganzzahligen Volumenverhältnissen erfolgen, zu einer Methode der Molekulargewichtsbestimmung aus.

$\mathrm{Zu}$ einer Routinemethode wurde die quantitative Elementaranalyse in der organischen Chemie jedoch erst, als es Liebig 1831 gelang, volumetrische und gravimetrische Verfahren zu einer leistungsfähigen und reproduzierbaren Methode zu verbinden. Sein bekannter »Kaliapparat« [32] ist ein miniaturisiertes Absorptionsgefäß, in dem Verbrennungsgase quantitativ aufgefangen und anschließend gewogen wurden. Damit konnten die Analysen nun auch von Hilfskräften erledigt werden, was kreatives Potential freisetzte und eine arbeitsteilige †Forschung ermöglichte. Dieser Vorgang der Verkapselung von Wissen und Prozeduren in die Black Box eines Instruments mit entsprechenden Rückwirkungen auf die Organisation der wiss. Arbeit wurde typisch für die Rolle von Instrumenten in der modernen Naturwissenschaft.

$\rightarrow$ Agrikulturchemie; Alchemie; Chemische Gewerbe; Metallurgie; Physikalische Wissenschaften; Wissen und Wissensideale

Quellen:

[1] H. DAVY, The Collected Works, Bd. 7, 1839-1840

[2] H. Kolbe, Ausführliches Lehrbuch der Organischen Chemie, 3 Bde., 1854-186o [3] C. H. Schreger, Kurze Beschreibung der technisch-chemischen Geräthschaften älterer und neuerer Zeit, 3 Bde., 1802 [4] G.F. Venel, Chymie, in: D. Diderot / J. D'Alembert, (Hrsg.), Encylopédie ou dictionnaire raisonné des sciences, des arts et métiers 3, 1753, 420-437

[5] J.G. Wallerius, Bref om Chemiens rätta Beskaffenhet, Nytta och Wärde, 1751.

\section{Sekundärliteratur:}

[6] F. Abbri, Science de l'air. Studi su Felice Fontana, 1991 [7] B. Bensaude-Vincent / F. Abbri (Hrsg.), Lavoisier in European Context: Negotiating a New Language for Chemistry, 1995 [8] B. Bensaude-Vincent / I. Stengers, Histoire de la chimie, 1993 [9] M. Beretta, The Enlightenment of Matter: The Definition of Chemistry from Agricola to Lavoisier, 1993 [10] M. Boas Hall, Robert Boyle and Seventeenth-Century Chemistry, 1958 [11] W.H. Brock, Viewegs Geschichte der Chemie, 1997 [12] M.P. Crosland, Historical Studies in the Language of Chemistry, 1962 [13] A. G. Debus, The Chemical Philosophy: Paracelsian Science and Medicine in the Sixteenth and Seventeenth Centuries, 1977 [14] J. GoLINSKI, Science as Public Culture: Chemistry and Enlightenment in Britain, 17601820, 1992 [15] H. Guerlac, The Chemical Revolution: A Word from Monsieur Fourcroy, in: Ambix 23, 1976, 1-4

[16] O. Hannaway, The Chemists and the Word: The Didactic Origins of Chemistry, 1975 [17] C. R. HiLL, The Iconography of the Laboratory, in: Ambix 22, 1975, 102-110 [18] F. L. Holmes, Lavoisier and the Chemistry of Life: An Exploration of Scientific Creativity, 1985 [19] E. Homburg, Van beroep >Chemikers: De opkomst van de industriële chemicus en het polytechnische onderwijs in Duitsland, 1790-1850, 1993 [20] K. Hufbauer, The Formation of the German Chemical Community, 17201795, 1982 [21] A.I. Ihde, The Development of Modern Chem- 
istry, 1964 [22] D. KNIGHT / H. Kragh (Hrsg.), The Making of the Chemist: The Social History of Chemistry in Europe, 17891914, 1998 [23] C. Meinel, Reine und angewandte Chemie: Die Entstehung einer neuen Wissenschaftskonzeption in der Chemie der Aufklärung, in: Berichte zur Wissenschaftsgeschichte 8, 1985, 25-45 [24] C. Meinel, Zur Sozialgeschichte des chemischen Hochschulfaches im 18. Jh., in: Berichte zur Wissenschaftsgeschichte 10, 1987, 147-168 [25] B. Meitzner, Die Gerätschaft der chymischen Kunst: Der Traktat \De sceuastica artis` des Andreas Libavius von 1606, 1995 [26] P. Ramberg, The Death of Vitalism and the Birth of Organic Chemistry: Wöhler's Urea Synthesis and the Disciplinary Identity of Organic Chemistry, in: Ambix 47, 2000, 170-195 [27] A.J. Rocke, Chemical Atomism in the Nineteenth Century, 1984 [28] J. G. SMITH, The Origins and Early Development of the Heavy Chemical Industry in France, 1979 [29] R. W. Sokup / H. Mayer, Alchemistisches Gold - Paracelsistische Pharmaka: Laboratoriumstechnik im

16. Jh. Chemiegeschichtliche und archäometrische Unter-

suchungen am Inventar des Laboratoriums von Oberstockstall/ Kirchberg am Wagram, 1997 [30] E. STRÖKER, Theoriewandel in der Wissenschaftsgeschichte: Chemie im 18. Jh., 1982

[31] F. Szabadváry, Geschichte der analytischen Chemie, 1966 [32] M.C. Usselman et al., Restaging Liebig: A Study in the

Replication of Experiments, in: Annals of Science 62, 2005, 1-55.

Christoph Meinel 